other process is needed to close the chain and allow for self-excitation. It is tempting to assume a certain flow pattern that twists the toroidal field in such a way that the net effect is a meridional component of the field. This leads to the formulation of the kinematical dynamo problem: Can we find a velocity field $\mathbf{v}(\mathbf{x}, t)$ in such a way, that the electrical field $\mathbf{v} \times \mathbf{B} / c$ drives a current $\mathbf{j}$ which amplifies the magnetic field $\mathbf{B}$ ?

In 1955, E.N. Parker presented a conceptual picture of a possible dynamo process based on the influence of rotation on convective motions due to the Coriolis force. F. Krause, K.H. Rädler and M. Steenbeck gave a more firm basis to it in the framework of the electrodynamics of mean fields. It has become clear since then that the correlation between velocity and vorticity, called "helicity", is the key parameter determining the possibility of dynamo action of a flow. Helicity can be understood as a "rest of order" in an otherwise chaotic flow. Helical flows such as turbulent convection under the influence of a Coriolis force are able to sustain a dynamo and build possible candidates for the "missing link" of the solar cycle, producing meridional field out of toroidal field.

Furthermore, turbulent flows in some cases are shown to enhance the diffusion and dissipation of a mean magnetic field through the formation of structures with small typical length scale, leading to a reduction of the effective electrical conductivity for the mean field.

The observed fact that stellar activity increases with the rate of rotation lends some support to the idea of turbulent dynamo action because the efficiency of the process increases with angular velocity as well. Model calculations incorporating the combined effects of differential rotation and helical flows display cyclic behaviour and can reproduce the basic features of the evolution of the large scale magnetic fields during the cycle: reversal of the polar fields, migration of the activity belt towards the equator and polarity rules.

\section{Tentative Conjecture}

In view of all the uncertainties mentioned in the introduction, a coherent theory of the physics of the solar cycle has not been given until now. But in order not to leave the reader alone with a bundle of concepts we give a conjecture (see Fig. 2) of how the solar cycle could operate, a picture presently shared by at least a fraction of active workers in the field.

Consider the transition region between the solar core and the convective zone at a depth of $\sim 200000 \mathrm{~km}$ from the surface. The observations of solar oscillations indicate that this zone is also a shear region between the slowly rotating convection zone and the much faster rotating solar core. This differential rotation produces a toroidal field, which is kept down by topo-

Fig. 2 - A possible sequence of processes related to the origin of the solar cycle

\begin{tabular}{|c|c|c|}
\hline Region & Physical Description & $\begin{array}{l}\text { Processes Related to Magnetic Field and } \\
\text { Activity Cycle }\end{array}$ \\
\hline Interface Zone & $\begin{array}{l}\text { Transition between } \omega_{\text {(core) }} \text { and } \\
\omega_{(\text {envelope) }} \text { Shear zone with pos- } \\
\text { sible turbulence driven by instabi- } \\
\text { lities due to differential rotation. }\end{array}$ & $\begin{array}{l}\text { Generation of toroidal field by differential rota- } \\
\text { tion. Field is kept down by topological pump- } \\
\text { ing until it has grown strong enough to escape } \\
\text { owing to buoyancy and magnetic instabilities; } \\
\text { formation of flux tubes. Helical flows produced } \\
\text { in that phase regenerate the poloidal field. }\end{array}$ \\
\hline Convection Zone & $\begin{array}{l}\text { Nearly adiabatically stratified re- } \\
\text { gion. Energy is transported almost } \\
\text { entirely by convection. The veloci- } \\
\text { ty field may consist of large-scale } \\
\text { eddies as well as small-scale tur- } \\
\text { bulence. }\end{array}$ & $\begin{array}{l}\text { Flux tubes are transported, shredded and refor- } \\
\text { med by convection and buoyancy. Small frag- } \\
\text { ments can be carried around by convection and } \\
\text { appear later as small active regions randomly } \\
\text { distributed over the surface. }\end{array}$ \\
\hline $\begin{array}{l}\text { Uppermost } \\
\text { Convection Zone } \\
\text { and Photosphere }\end{array}$ & $\begin{array}{l}\text { Transition between energy trans- } \\
\text { port by convection and radiation. } \\
\text { Stratification is slightly super- } \\
\text { adiabatic in the upper convection } \\
\text { zone and sub-adiabatic in the pho- } \\
\text { tosphere. Velocity field consists of } \\
\left.\text { supergranulation (scale } \sim 10^{4} \mathrm{~km}\right) \text {. } \\
\text { granulation }\left(\sim 10^{3} \mathrm{~km}\right) \text { and small- } \\
\text { scale turbulence. }\end{array}$ & $\begin{array}{l}\text { Flux tubes suffer from local instabilities leading } \\
\text { to loop formation, break through the photo- } \\
\text { sphere and build active regions. Adiabatic } \\
\text { downflow along the loops cools relative to the } \\
\text { superadiabatic exterior and leads to an even } \\
\text { stronger concentration of fields. Sunspots ap- } \\
\text { pear and are eventually disrupted by velocity } \\
\text { fields. }\end{array}$ \\
\hline
\end{tabular}

logical pumping until it has grown strong enough to escape by means of magnetic buoyancy or a related instability. Helical flows produced in that phase by the influence of the Coriolis force on the velocity field possibly close the dynamo chain and regenerate the meridional field. After the field has left the amplification region, it is lifted upwards by the combined effects of convection and buoyancy in the form of concentrated flux tubes which break through the surface eventually and build active regions of all sizes. In the meantime, differential rotation is already producing the toroidal field for the next half cycle from the regenerated poloidal field.

\section{REFERENCES}

The interested reader is referred to: 1. Krause F. and Rädler K.H., Mean-Field Magnetohydrodynamics and Dynamo Theory, (Pergamon Press, Oxford), 1980.

2. Moffatt H.K., Magnetic Field Generation in Electrically Conducting Fluids, (Cambridge University Press, Cambridge) 1978.

3. Parker E.N., Cosmical Magnetic Fields, (Clarendon Press, Oxford) 1979.

4. Stix M., "Theory of the Solar Cycle", Solar Physics 74 (1981) 79-101.

5. Piddington J.H., "Solar Magnetic Fields and Convection. VII. A Review of the Primordial Field Theory", Eds. V. Bumba and J. Kleczek, Proc. IAU - Symp. 71 Basic Mechanics of Solar Activity, (Dordrecht) 1976, p.389.

\title{
Physics of the Solar Corona
}

\author{
Claudio Chiuderi, Florence \\ (Istituto di Astronomia, Università di Firenze)
}

The solar corona is the outermost part of the atmosphere of the Sun, a tenous, hot plasma extending outwards from slightly above the visible limb to well beyond the Earth's orbit. The denser part of this plasma, close to the Sun's surface, forms the corona proper, only visible during total solar eclipses or by means of special instruments, the coronagraphs, at least at optical wavelength. Typical values of the physical corona parameters are a temperature of the order of $1-2 \times 10^{6} \mathrm{~K}$, with occasional localized increases up to a few times $10^{7} \mathrm{~K}$, and a number density in the range $10^{8}-10^{10} \mathrm{~cm}^{-3}$.

Because of the difficulty of observing optically the solar corona, a rapid development did not take place until observations at different wavelengths made this part of the atmosphere more accessible. From the typical values quoted above, it is clear that the corona will emit at radio wavelengths as well as in the X-ray region. The terrestrial atmosphere is transparent to radio waves and opaque to $X$-rays, so that while coronal observations at radio frequencies can be made routinely, X-ray observations can be only performed from space, and our information is necessarily more fragmented in time.

The corona is in a state of continual expansion, the consequent outflow of matter constituting the solar wind, with an average flux of $3 \times 10^{-16} \mathrm{~g} \mathrm{~cm}^{-2} \mathrm{~s}^{-1}$, which corresponds to a total mass loss of $\sim 10^{-14}$ solar masses per year. The properties of the solar wind have been studied in situ by artificial satellites from about $0.3 \mathrm{AU}$ to $1 \mathrm{AU}$ (1 $\mathrm{AU} \cong 150 \mathrm{Mkm}$ ).

There are many reasons for our interest in coronal studies: the Sun is the only star in our vicinity, and the solar corona can be considered a giant plasma laboratory, 


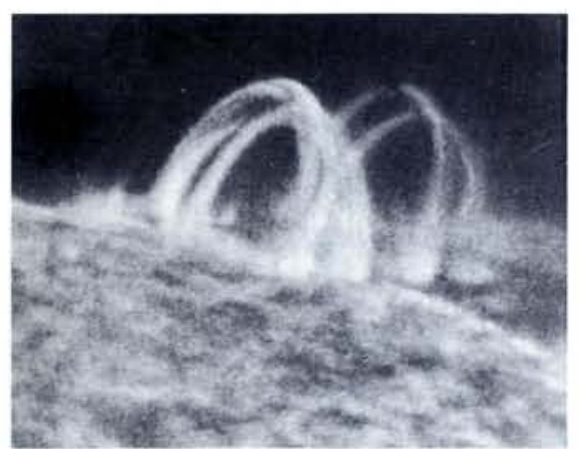

A spectacular example of solar corona loops.

where experiments are continuously being performed under conditions that are ureproducible in terrestrial laboratories. Although very hot plasmas are currently produced on Earth, their spatial and temporal scales are so vastly different from those encountered in space, extrapolation becomes unsafe. Conversely, plasma physics in the laboratory has a lot to learn from astrophysical plasmas and the close connection between the two has drawn many astrophysicists and plasma physicists together to start an extremely fruitful collaboration.

\section{Coronal Heating}

One of the main problems of coronal physics that is still unresolved, is that of coronal heating. Whereas the corona has a temperature of the order of millions of degrees, the underlying atmospheric layers have a much lower temperature. The photosphere, namely the region where the visible light is emitted, has a temperature of $\sim 5000 \mathrm{~K}$. It is clear therefore, that the corona cannot be heated radiatively and that some heating mechanism must be operating to create and maintain the corona in its observed state.

At its inner boundary, the corona terminates rather abruptly, with the temperature falling precipitously to chromospheric values ( $\cong 10^{4} \mathrm{~K}$ ) over a distance of about $100 \mathrm{~km}$, giving rise to a very steep temperature gradient. In this thin transition region, the density decreases rapidly in the outward direction, so that the pressure remains approximately constant. The existence of a large temperature gradient, coupled with the high thermal conductivity of the solar plasma implies that a large amount of heat is continuously flowing from the corona to the lower atmosphere, and this loss; together with that due to radiation and to the outward convection related to the solar wind, must be constantly replenished.

A clue to the answer to this problem is given by considering the thermal stability of a radiative atmosphere. The global radiative losses, $Q$, of an optically thin plasma at a temperature of $10^{4} \mathrm{~K} \leq T \leq 10^{8} \mathrm{~K}$ can be put in the form:

$$
Q=\rho^{2} \phi(T)
$$

where $\rho$ is the density and $\phi(T)$ can be computed from the knowledge of composi- tion of the solar plasma. $Q$ includes both the contribution of the continuum and that of the lines coming from partially ionized elements like $\mathrm{C}, \mathrm{O}, \mathrm{N}, \mathrm{Ne}, \mathrm{Mg}, \mathrm{Fe}$ etc. The point of interest here is that $\phi(T)$ is a decreasing function of the temperature in the range $10^{5} \mathrm{~K} \leq T \leq 10^{7} \mathrm{~K}$. Thus a plasma with a temperature in this interval will react to a cooling perturbation by increasing its radiative output, which in turn will decrease the temperature still further. In other words, a homogeneous plasma would be unstable to thermal instabilities: direct computations show that the final equilibrium will be one with a thin transition layer separating a high temperature region from a low temperature one, as found in the coronal structure.

As the behaviour is quite insensitive to changes in the heating mechanism that is assumed to be operating in the upper atmospheric layers, no information on the nature of the heating processes can be inferred from the temperature structure alone. Various attempts to identify the physical process, or processes, have been made during the past years, but none has convincingly proved the dominance of one process over the many others that can be proposed. There are however, a certain number of conditions that must be satisfied by every acceptable model.

In the first phase, the energy source must lie below the corona and most likely below the photosphere. This because the state of the corona reflects closely that of the underlying regions of the atmosphere and in particular is intimately connected with the various phenomena normally indicated as solar activity. This energy must be propagated upwards and must be deposited in the appropriate amount in the corona. There are many possible schemes that fulfill the above conditions. For instance, acoustic noise can be generated by the turbulent motions known to exist in the subphotospheric layers, the energy from which would be carried outwards by waves. In principle, in a compressible atmosphere embedded in a gravitational field, there are two fundamental wave modes, the acoustic mode and the internal gravity mode, according to the nature of the restoring force, compressibility and buoyancy respectively. The acoustic mode seems to be the most likely candidate. These sound waves propagate in a medium of decreasing density and eventually steepen into shocks, depositing a fraction of their energy through the dissipative processes operating in the shock front.

This type of theory encounters a number of problems. First of all a large fraction of the energy is reflected downwards by the strong density gradient encountered, so that it is doubtful if the required amount of the energy will eventually reach the corona. Second, this theory applied to stars, different from the Sun, fails to explain the characteristics of stellar coronae recently observed in $\mathrm{X}$-rays from the satellite EINSTEIN.

It is not difficult to identify the probable cause of this failure. We have so far neglected mentioning one essential ingredient of the solar corona: the magnetic field and this plays a vital and unique role in coronal physics. If heating were to be produced purely by acoustic shocks, the relationship with magnetic properties would be

\title{
NOR DITA nORDisk institut for teoretisk ATOMFysik NORDI A Danmark · Finland · Island · Norge · Sverige
}

\section{Assistant Professorship in Theoretical Astrophysics}

NORDITA, the Nordic theoretical physics institute located at the Niels Bohr Institute of Copenhagen University, has an opening starting September 1982 for a theoretical astrophysicist. The initial appointment will be for three years, with the possibility of renewal up to a total of six years. Salary will be in the range of $170,000-250,000$ Danish Kroner depending on experience. The successful applicant is expected to guide fellows at roughiy the post-doctoral level and to interact with colleagues at NORDITA and elsewhere in the Nordic countries. This position provides excellent opportunities to pursue original research, to travel to other institutes and to meetings, and to invite guest scientists. There is no restriction on the applicant's field of research, though preference will be given to those areas where physics and astrophysics are most closely coupled. Recent research activities at NORDITA have involved neutron stars, supernovae, X-ray sources, cosmic rays and the formation of galaxies.

Send before 15 March, a curriculum vitae, list of publications (with titles) and the names of at least three referees to

\author{
N.R. Nilsson, NORDITA, Blegdamsvej 17 \\ DK-2100 Copenhagen O, Denmark
}

There is no restriction as to the sex or nationality of the applicant. 
a very loose one, contrary to what is observed.

\section{Magnetic Influences}

The presence of magnetic fields on and above the solar surface is a well-established fact. Sunspots are known to possess fields in the kilogauss range, eclipse observations show clearly a shaping of the corona reminiscent of magnetic structures, and magnetic fields are carried along by the solar wind where they are directly measured. Fields at the photospheric level can be inferred from the Zeeman splitting of spectral lines. No direct measurement of corona fields is available so far and does not appear likely in the near future. From the measured photospheric line-of-sight fields, however, it is possible, under given assumptions, to extrapolate a three-dimensional structure of the solar magnetic field at higher levels. These extrapolations present a striking similarity with the emission features observed in the $\mathrm{X}$-ray wavelength range in a number of space experiments and especially during the three Skylab Missions in 1972-73.

These observations proved that the corona is far from being homogeneous and actually presents such dramatic differences from one region to another that it appears to be a collection of widely different entities. The X-ray emission is concentrated in bright structures that have the form of arches (coronal loops) which group together to form the so-called active regions. Contrasting with these bright areas, there are regions of very reduced emission (coronal holes) that are typically found near the solar poles, but may occasionally extend to the equator or even form an elongated dark strip along the meridian connecting one polar region to the other.

All these coronal structures are in a very dynamic state. They appear, evolve, dissapear over a large variety of timescales from minutes to months. From the extrapolations of photospheric fields we see that apart from their intrinsic emissivity, loops and holes differ also for the topology of the associated magnetic fields, coronal holes corresponding to open and coronal loops to closed field structures. There are also clear indications that the solar wind, or at least the high speed streams, flows preferentially from coronal holes.

The extreme structuring of the solar corona suggests that rather than considering the properties of the solar corona on a global scale, i.e. averaging over the individual structures, one should study the properties of the single constituents. This explains why most of the recent work has concentrated on the properties of the coronal loops. They form a subset of the corona for which a large amount of high quality observational material is available and seem to be amenable to a rather detailed theoretical investigation. They are also the seat of coronal flares pictorially described as explosions in a magnetized plasma. The plasma physics of coronal loops poses a variety of exciting and subtle problems.

\section{A Composite View}

Let us however return to the problem of coronal heating and include the magnetic field in our basic parameters. The solar plasma is highly conducting and thus the field lines are tied up with matter. However in the photosphere, the gas pressure exceeds the magnetic pressure (the plasma parameter $\beta>1$ ) whereas the opposite is true in the corona. Turbulent movements at the photospheric levels, induce magnetic stresses that propagate upwards where they can relax, giving up the magnetic energy associated with them. The propaga- ting magnetic stresses can be alternatively described as magnetohydrodynamic waves (instead of the acoustic waves previously considered) or as currents. The study of the transformation of magnetic energy into heat and bulk motion of particles constitutes one of the main objectives of modern coronal physics.

\section{SUGGESTED READING}

1. Vaiana G.S. and Rosner R., "Recent Advances in Coronal Physics", Annual Review of Astronomy and Astrophysics, 16 (1978) 393.

2. Solar Phenomena in Stars and Stellar Systems, Eds. R.M. Bonnet and A.K. Dupree, NATO Advanced Study Institutes Series, (Reidel Publ. Co., Dordrecht) 1981

3. Solar Flare Magnetohydrodynamics, Ed. E.R. Priest, (Gordon and Breach Science Publ., New York) 1981.

\title{
Magnetic Face of the Sun
}

\author{
Cornelis Zwaan, Utrecht \\ (Sterrewacht "Sonnenborgh")
}

The investigation of sunspot physics started with the application of spectroscopy and polarimetry by Hale beginning in 1905 at Mt. Wilson. Hale found that sunspots are much cooler than the ambient solar atmosphere. Furthermore he detected the presence of strong magnetic fields from the Zeeman effect in sunspot spectral lines.

The strong magnetic field is the basic property of sunspots. In the dark inner part, called the umbra, (Fig. 1), the field is nearly vertical; across the surrounding penumbra the field is inclined progressively, becoming nearly horizontal at the outer edge. Thus the field resembles the magnetic field near the end of a long solenoid lying vertically below the sunspot.

The field intensity $B$ measured in the umbral cores is 0.25 to $0.33 \mathrm{~T}$ in large spots, and $0.19<B<0.25 \mathrm{~T}$ in the smallest spots and pores.

There is magnetic flux outside sunspots as well, in discrete elements (faculae) arranged in patterns called facular plages and the network (Fig. 2). In white light these faculae are not conspicuous near the centre of the solar disk, but they stand out as bright features near the solar limb. They are bright all over the disk (Fig. 2c) in the cores of strong spectral lines formed at larger heights.

Since 1966, indications have been accumulated which lead to the conclusion that outside sunspots, the magnetic field also consists of discrete elements of high intensity: $0.10<B<0.20 \mathrm{~T}$, separated by an atmosphere without a measurable magnetic field. The observational proofs did not come easily, as the magnetic elements outside sunspots are quite small: their diame- ters are $400 \mathrm{~km}$ or less, just below the resolution limit of ground-based telescopes during the best observing conditions. Moreover, the "filling factor" of magnetic structure is small: in the photosphere, which is the deep atmosphere where the bulk of the optical radiation originates, the magnetic structure occupies at most $20 \%$ in facular plages in the vicinity of sunspots (see Fig. 2), and much less than $1 \%$ in the "quiet" photosphere.

Thus the Sun faces us with a hierarchy of discrete magnetic elements, ranging from large sunspots (diameter $2 R \cong 55000$ $\mathrm{km}$, flux $\Phi \cong 3 \times 10^{14} \mathrm{~Wb}$, central field intensity $B \cong 0.30 \mathrm{~T}$ ), through dark pores $\left(2 R \cong 3500 \mathrm{~km}, \Phi \cong 2 \times 10^{12} \mathrm{~Wb}, B \cong\right.$ $0.23 \mathrm{~T})$, to small bright elements $(2 R \cong 300$ $\mathrm{km}, \Phi \cong 1 \times 10^{10} \mathrm{~Wb}, B \cong 0.15 \mathrm{~T}$ ). These elements are present for both polarities, and they are distributed over the solar disk in complicated and irregular patterns. Moreover, major changes in the field pattern occur within a few months. Thus the solar magnetic field is utterly different from the smooth dipole-like field of the Earth.

We now discuss the physics of the individual magnetic elements in terms of simple fluxtube models. These models are based on the following constraints (see Fig. $3)$ : The fluxtube is vertical and rooted in the convection zone, which extends from the bottom of the photosphere down to a depth of $2 \times 10^{5} \mathrm{~km}$ ( $\cong 1 / 3$ solar radius) in the solar interior (see Gough page 4). The absence of large mass velocities suggests a static mechanical equilibrium: the vertical component of the pressure gradient is balanced by gravity, and the horizontal component by the Lorentz force. 